\title{
Research article \\ Efficacy of motor therapy over sensorimotor therapy in upper limb functions of post stroke patients
}

\author{
V. Rajalaxmi ${ }^{1}$, Tharunya M. ${ }^{2}$, S. S. Subramanian ${ }^{3}$, G. Vaishnavi ${ }^{4}$, R.N.V. Deepthi ${ }^{4}$, Chandra Kumar K ${ }^{4}$, N.Sukala ${ }^{4}$, \\ K.Balathandayutham ${ }^{5}$
${ }^{1}$ Vice Principal, Faculty of Physiotherapy, Dr. M.G.R. Educational and Research Institute University, Velappanchavadi, Chennai - 600 077, Tamil Nadu, India
${ }^{2}$ Physiotherapist, Freelancer, Chennai, Tamil Nadu, India
${ }^{3}$ Principal, Balaji College of Physiotherapy, Bharath University, Chennai, Tamil Nadu, India
${ }^{4}$ Faculty of Physiotherapy, DRMGRERI, ${ }^{5}$ Lecturer, Annamalai University Chidambaram, Tamil Nadu, India

(Received: August $2020 \quad$ Revised: March $2021 \quad$ Accepted: March 2021)

Corresponding author: V. Rajalaxmi. Email: rajalaxmiphysio@gmail.com

\begin{abstract}
Introduction and Aim: Stroke is one of the leading causes of death and disability in India. The incidence of stroke rapidly increases with age, doubling for each decade after 55. In the majority of patients the upper limb is severely impaired than lower limb. The aim of this study was to analyze the efficacy of motor therapy over sensorimotor therapy in upper limb functions of post stroke patients.

Materials and Methods: This was an experimental study, of pre-post type, that was conducted in the outpatient department of Physiotherapy at ACS Medical College and Hospital,chennai.30 samples were selected from 45 volunteers according to inclusion criteria and the study was conducted for Group A received sensorimotor therapy for one session/day for 6 days a week for 12 weeks. Group B received motor therapy for one session/day for 6 days a week for 12 weeks. Pre and post measurement were done using motor assessment scale, stroke upper limb capacity scale, action research arm test and nine-hole peg test.
\end{abstract}

Results: There was a significant difference in mean values of group A and B on comparison. Group A seems to be more effective than group B.

Conclusion: The study concludes that both motor therapy and sensorimotor therapy are beneficial in stroke rehabilitation, but sensorimotor therapy is more effective in improving the gross and fine motor skills of the upper limb of stroke patients.

Keywords: Sensorimotor therapy; motor therapy; post stroke.

\section{INTRODUCTION}

$\mathrm{T}$ he world health organization (WHO) defined stroke as rapidly developing clinical signs of focal disturbance of cerebral function with symptoms lasting 24 hours or longer or leading to death with no evident cause other than vascular origin. Among the entire causes of death stroke ranks 5. Globally there were 6.5 million stroke deaths making stroke the second leading cause of death (1). The prevalence rate that is estimated for stroke ranges from $84-262 / 100,000$ in rural and 334-424/100,000 in urban areas. The incidence rate is $119-145 / 100,000$ based on the recent population studies in 2017 (2). Globally, $90.5 \%$ of the stroke burden was attributable to the modifiable risk factors including smoking, diet and low physical activity (3). The principal cause of disability in Brazil is stroke. The world health organization estimates that stroke events in EU countries are likely increasing by $30 \%$ between 2000 and 2025 . The most common deficit after stroke, which is seen with more than $80 \%$ of stroke patients is contralateral upper limb hemiparesis (4).

Marini et al, who analyzed 29 studies including 3,589 patients less than 45 years of age with first-ever stroke, published between 1980 and 2009, published a systematic review on the incidence of stroke in young adults. Rough rates span from 5.76/100,000 to $39.79 / 100,000$ and standardized rates spans from $6.14 / 100,000$ to $48.51 / 100,000$. In the same review, the proportion of ischemic strokes ranged between $21.0 \%$ and $77.9 \%$, intracerebral hemorrhage between $3.7 \%$ and $38.5 \%$, and subarachnoid hemorrhage between $9.6 \%$ and $55.4 \%$. The current study from Bosnia and Herzegovina states that $61 \%$ of the cases among young adults have ischemic stroke, $17 \%$ have intracerebral hemorrhage, and $22 \%$ subarachnoid hemorrhage (5). Due to various reasons the comparison between the study are difficult and reasons include the methodology used, age groups, diagnostic criteria, time scale, and a change in the pathology. The percentage of first-ever strokes in young adults varies from country to country, and spans from $5-20 \%$ (6). Persistent atrial fibrillation is the potent risk factor for first and recurrent stroke (7).

Myocardial Infarction commonly causes intraventricular thrombus to form on the damaged surface of the endocardium. Stroke can occur in up to $12 \%$ of patients with acute myocardial infarction and a 
left ventricular thrombus (8). Atrial Fibrillation with mitral valve disease has been considered as a risk factor for stroke (9). Venous thrombo emboli from leg to pelvic vein enter the right atrium and cause the blockage of heart finally leading into cerebrovascular arterial occlusion causing stroke (10). The common pathophysiology of stroke is lack of sufficient blood flow to perfuse cerebral tissue as a result of narrowing or blockage of arteries supplying brain (11). Narrowing of blood vessels is the result of atherosclerotic plaque formation and can cause a blood clot to form in the blood vessels leading to stroke (12). In the ischemic core area of stroke, blood flow is so drastically reduced that cells usually cannot recover and subsequently leads to cellular death (13). Blood clots or debris from elsewhere in the body typically the heart a valve travel through the circulatory system and finally blocks the cerebral blood vessels (14).

Stroke may lead to both structural and functional types of impairments. Upper limb impairments after stroke are the cause of functional limitations associated with usage of the affected upper limb. An accurate knowledge on underlying impairments is essential to provide appropriate treatment. Recognition of underlying impairments of hand is difficult due to two reasons.1) The impairments are not stable i.e. as motor recovery advances, the type as well as nature of the impairments may vary: therefore, the treatment needs to be changed to focus on the impairment leading to dysfunction at a given point in time. 2) There are chances of multiple impairments presenting at a time, i.e a patient may have weakness of the arm and hand, along with spasticity following a stroke that makes it confusive whether to treat spasticity or weakness first. (15).

After a central nervous system injury, neural reorganization serves as the foundation for learning, the acquisition of new skills and the basis of recovery of function (16). Adaptation of neural system promotes powerful recruitment of motor neuron pools, new synapse evolution, transfer of function from impaired areas to preserved areas, strengthening of parallel synapses, increased dendritic sprouting, enhanced myelination of remaining neurons or modification of cortical and non-cortical representations (17-19).

One of the commonly used preventive and rehabilitative exercise techniques in rehabilitation settings is sensorimotor training (20). Sensorimotor training uses some combination of sensory input and motor activities to facilitate the expected normal motor response, which is defined as behavior of a more advanced, organized, flexible nature than that occurred prior to stimulation. The sensorimotor tasks are texture discrimination, limb position sense, and tactile object recognition. Motor learning is considered crucial for rehabilitation in general. In stroke, motor learning does not refer to the acquisition of new skills, but to the relearning process of previously acquired movement patterns (21). Individually tailored motor therapy consists of a unilateral motor exercise program for the affected upper limb, exercises are basic shoulder level exercises such as towel slide, shoulder shrug, external rotation with cane, behind the neck cup pass and therapeutic ball exercises for hand recovery (22). Neuro developmental treatment (NDT) is considered one of the most common neurophysiological treatment approaches in contemporary post stroke rehabilitation (23). It is not specifically a set of exercises but rather a task-oriented training that incorporates knowledge in motor learning, motor control, biomechanics, muscle physiology and activity - dependent brain plasticity mediating recovery after a brain lesion. It allows for adaptive and anticipatory mechanisms (24).

PNF is a neurophysiological approach that uses impulses from the periphery to stimulate the central nervous system by facilitation of sensory receptors present in muscles and around the joints by stretch, resistance, traction, approximation, audiovisual command and manual contact to the patient. PNF is popularly used in clinical settings to improve both active as well as passive range of motion with endmost aims being to optimize motor performance and rehabilitation (25). Proprioceptive Neuromuscular Facilitation techniques assist in improving muscular strength and endurance, joint stability, mobility, neuromuscular control and coordination,thereby leading to maximum functional performance.

The ARAT has been found useful in prior studies evaluating stroke patients across a wide spectrum of impairments. The test shows good validity, as well as sensitivity to spontaneous and therapy-related gains after stroke. Interrater and intrarater reliability have been reported to be high; however, each of these values represents reliability as assessed within a single institution. Increasingly, multisite trials of acute stroke have embraced the importance of reducing the interstice variance that is present when assigning a score for outcome assessments.

The 9HPT seems to be an essential and valid tool for making decisions about early intervention, which helps to reduce the severity of motor impairment and improve quality of life.

MAS to be useful for providing feedback to the patient on his progress, and it can be used to motivate him toward recovery. Two questionnaires are being designed to investigate whether progress measured by the MAS relates to the physical therapists and patient's subjective impressions of progress. We have developed this MAS for stroke patients and investigated it for reliability. This scale has been found to be highly reliable and its validity and usefulness in measuring the progress of patients in physical therapy should be investigated. Because the reliability of each individual rater is considered as an essential factor in the use of this scale, we recommend that physical therapists intending to use the MAS should become familiar with 
the criteria for scoring by testing at least six patients before formally using it in clinical practice.

SULCS is a novel and internally compatible, unidimensional scale that is used to assess upper limb capacity, in patients after stroke. It is a welldocumented and easy to use instrument in these days.

\section{MATERIALS AND METHODS}

This was an experimental study of pre-post type with 30 subjects. The study was carried out in ACS medical college and hospital and it took 3 months to complete the study (January 2019-April2019). Clinically diagnosed acute hemiplegic stroke patients within 8 weeks, between 50-60 years, and both male and female were included in the study. Pre and Post intervention findings of samples were assessed using the outcome measures NHPT, ARAT, MAS and SULCS.

Once the study got approval from the institutional review board (IRB), 30 subjects were selected from 45 volunteers depending on the inclusion criteria. Participants were divided into two groups by lottery method where, even numbers will be categorized in group $\mathrm{A}$ and odd numbers in group $\mathrm{B}$. The subjects were fully explained about the study and were asked to fill the consent form in acceptance to participate in the study, which is duly signed by the participants and therapist. Initially demographic details like age, gender, height, weight, were collected ensuring confidentiality of the same. Group A received sensorimotor therapy for one session for 6 days and for 12 weeks. Group B received motor therapy for one session for 6 days and for 12 weeks. After completing the study for three months, the posttest measurements were taken and compared with Pretest measurements by using Motor assessment scale, Stroke upper limb capacity scale, Action research arm test, Nine-hole peg test. Finally, the data were compared and analyzed with and between groups.

\section{Statistical analysis}

Inferential and descriptive statistics are used to tabulate and analyse collected data.All the parameters were assessed using the statistical package for social science (SPSS) version 20.0. Independent t-test (Student t-Test) was adopted to find statistical differences between the groups.

Table 1: Comparing the values of MAS between group A and group B in pre and post-test values using independent Student's ' $t$ ' test

\begin{tabular}{|c|c|c|c|c|c|c|c|c|c|}
\hline \multirow[t]{2}{*}{ Mas } & \multicolumn{2}{|c|}{ Group A } & \multicolumn{2}{|c|}{ Group B } & \multirow[t]{2}{*}{$\begin{array}{c}\text { T- } \\
\text { Test }\end{array}$} & \multirow[t]{2}{*}{ Df } & \multicolumn{2}{|c|}{$\begin{array}{c}\text { 95\% Ci Of The } \\
\text { Difference }\end{array}$} & \multirow[t]{2}{*}{$\begin{array}{c}\text { SIG (2- } \\
\text { tailed) }\end{array}$} \\
\hline & Mean & S.D & Mean & S.D & & & Lower & Upper & \\
\hline Pre Test & 3.13 & 0.91 & 1.80 & 0.77 & 4.306 & 28 & 0.69 & 1.96 & $0.000 * *^{*}$ \\
\hline Post Test & 7.86 & 0.99 & 5.2 & 0.77 & 8.214 & 28 & 2.0 & 3.33 & $0.000 * *^{*}$ \\
\hline
\end{tabular}

$(* * *-\mathbf{P} \leq 0.001)$

Table 2: Comparing the values of SULCS between group A and group B IN pre and posttest values using independent Student's ' $\mathrm{t}$ ' test

\begin{tabular}{|c|c|c|c|c|c|c|c|c|c|}
\hline \multirow{2}{*}{ Sulcs } & \multicolumn{2}{|c|}{ Group A } & \multicolumn{2}{|c|}{ Group B } & \multirow{2}{*}{ T-Test } & \multirow{2}{*}{$\begin{array}{c}\text { D } \\
\text { f }\end{array}$} & \multicolumn{2}{|c|}{$\begin{array}{c}\text { 95\% Ci Of The } \\
\text { Difference }\end{array}$} & \multirow{2}{*}{$\begin{array}{l}\text { SIG(2- } \\
\text { tailed) }\end{array}$} \\
\cline { 2 - 5 } \cline { 7 - 8 } & Mean & S.D & Mean & S.D & & & Lower & Upper & \\
\hline Pre Test & 3.66 & 1.11 & 2.8 & 0.83 & 2.228 & 28 & 0.06 & 1.53 & $0.340^{*}$ \\
\hline Post Test & 7.3 & 0.97 & 6.9 & 0.59 & 1.356 & 28 & -0.2 & 1.0 & $0.186^{*}$ \\
\hline
\end{tabular}

$(* *-\mathrm{P} \leq 0.005)$

Table 3: Comparing the values of NHPT between group A and group B in pre and post-test values using independent Student's ' $t$ ' test

\begin{tabular}{|c|c|c|c|c|c|c|c|c|c|}
\hline \multirow{2}{*}{ NHPT } & \multicolumn{2}{|c|}{ Group A } & \multicolumn{2}{|c|}{ Group B } & \multirow{2}{*}{ t-Test } & df & \multicolumn{2}{c|}{$\begin{array}{c}\text { 95\% CI of the } \\
\text { Difference }\end{array}$} & $\begin{array}{c}\text { SIG(2- } \\
\text { tailed) }\end{array}$ \\
\cline { 2 - 4 } & Mean & S.D & Mean & S.D & & & Lower & Upper & \\
\hline Pre Test & 91.77 & 3.62 & 90.7 & 5.43 & 0.617 & 28 & -2.41 & 4.49 & $0.409^{*}$ \\
\hline Post Test & 79.36 & 4.71 & 85.6 & 4.53 & --3.694 & 28 & -9.7 & -2.77 & $0.001^{*} *^{*}$ \\
\hline
\end{tabular}

$(* *-\mathrm{P} \leq 0.005)$

Table 4: Comparing the values of ARAT between group-a and group-b in pre and post test values using independent Student's ' $t$ ' test

\begin{tabular}{|c|c|c|c|c|c|c|c|c|c|}
\hline \multirow{2}{*}{ ARAT } & \multicolumn{2}{|c|}{ Group A } & \multicolumn{2}{|c|}{ Group B } & t-Test & df & \multicolumn{2}{c|}{$\begin{array}{c}\text { 95\% CI of the } \\
\text { Difference }\end{array}$} & $\begin{array}{c}\text { SIG(2- } \\
\text { tailed) }\end{array}$ \\
\cline { 2 - 11 } & Mean & S.D & Mean & S.D & & & Lower & Upper & \\
\hline Pre Test & 6.06 & 0.88 & 4.6 & 1.91 & 2.688 & 28 & 0.34 & 2.58 & $0.121^{*}$ \\
\hline Post Test & 12.3 & 1.23 & 8.1 & 1.40 & 8.689 & 28 & 3.21 & 5.1 & $0.000^{* *}$ \\
\hline
\end{tabular}

$\left(* *_{-} \mathrm{P} \leq 0.005\right)$ 


\section{RESULTS}

On comparing the mean values of MAS scores between the Group A and Group B, both the groups have showed improvement in the post test mean values although the group- A posttest mean value (7.86) showing lesser mean value is more effective than Group- B post-test mean value (5.2) and statistically significant at (*- $\mathrm{P}$ $<0.001)$. There is a significant difference in the pretest and the post-test values of MAS test scores between the Group A and Group B. Hence, the null hypothesis is rejected.

On comparing the mean of SULCS test between the Group A and Group B, both the groups showed increased SULCS in the posttest mean values. But the Group A posttest mean value (7.3) showing the higher mean value is more effective than Group B post-test mean value (6.9) and statistically significant at (*-P < $0.001)$. There is a significant difference in the pretest and posttest values of SULCS between the Group A and Group B. Hence, the null hypothesis is rejected.

On comparing the mean of NHPT test between the Group A and Group B, both the groups showed increased NHPT in the posttest mean values. But the Group A posttest mean value (79.36) showing the lower mean value is more effective than Group B posttest mean value (85.6) and statistically significant at $\left(*_{-} \mathrm{P}<0.001\right)$. Furthermore, there is a significant difference in the pretest and posttest values of NHPT between the Group A and Group B. Hence, the null hypothesis is rejected.

On comparing the mean of ARAT test between the Group A and Group B, both the groups showed increases in the posttest mean values. But the Group A posttest mean value (12.3) showing the higher mean value is more effective than Group B posttest mean value $(8.1)$ and statistically significant at $\left(*_{-} \mathrm{P}<0.001\right)$. In addition, there is a significant difference in the pretest and posttest values of ARAT between the Group A and Group B. Hence, the null hypothesis is rejected.

\section{DISCUSSION}

The present study was conducted with the sample size of 30 subjects to find out the efficacy of motor therapy over sensorimotor therapy in upper limb function of post stroke patients. Subjects included in the study received allocated motor therapy or sensorimotor therapy for the duration of 12 weeks and found that there is an improvement in all the aspects of motor and sensory between both the groups. Somatosensory function is reported to be associated with motor and functional outcome after stroke and somatosensory impairment is reported to have a negative influence on motor recovery. Recently only two studies that focus on sensorimotor training in motor function were identified. Both studies reported improvement in motor function after sensorimotor training. Our attempt with an RCT design will provide additional information regarding the clinical effect of sensorimotor training on motor as well as somatosensory function. Despite the strengths, some challenges remain in our study. Good clinical assessments for motor and somatosensory function often lack sensitivity to change. We put together an extensive and well-established assessment battery of tests including both function and activity level. Both assessments of upper limb motor (FMA) and somatosensory (Em-NSA) function as well as more specific assessments for dexterity (9HPT), proprioceptive (WPST), or touch discriminative function (TDT) will be combined in the protocol to cover a broad spectrum of somatosensory and motor function, and activity level performance of patients.

Sensitivity is addressed using the addition of perceptual threshold of touch as an outcome measure for light touch. The consistency of intensive movement training protocols with respect to current health care delivery models will require an economic analysis to overcome program inertia. Weakness of the upper extremity may be associated with other neurological features that affect the recovery of motor function and that requires additional and targeted therapeutic intervention.

Prevalence rate for deficits in somatic sensation following stroke spans from 12-15\%. Functionally, the motor problems resulting from sensory deficits after stroke can be summarized as impaired detection of sensory information, disturbed motor tasks performance requiring somatosensory information, and diminished upper extremity rehabilitation outcomes. Even Though patient has adequate motor recovery, sensory improvement is must for safety. Also, up to $50 \%$ of patients experience pain of the upper extremity during the first year after stroke, especially shoulder pain and complex regional pain syndrome-type I (CRPS- type I), which may impede adequate early rehabilitation, since it is a more objective and a ratioscaled outcome measure.

Assessment of somatosensory function may be challenging in patients with communication difficulties. In addition, attention deficits have significant negative influence on somatosensory assessment. The reduction in somatosensory function along with lack of attention to the somatosensory stimuli during evaluation can lead to patients incorrectly reporting not to feel the stimuli. We assume that brain connectivity changes are correlated to recovery of sensorimotor function and better normalization of sensorimotor networks are found for the sensorimotor therapy group.

Second, with this randomized controlled trail (RCT), we investigate the effect of a novel sensorimotor program on upper limb function. To our knowledge, this kind of therapy is under investigated. Somatosensory function is reported to be associated with motor and functional outcome after stroke and somatosensory impairment is reported to have a negative influence on motor recovery. To date, only two studies investigating a sensorimotor program on 
motor function are reported. Both studies were case series but did report improved scores on motor function after training. Our trial with an RCT design will provide additional knowledge concerning the clinical effect of sensorimotor training on motor as well as somatosensory function.

\section{CONCLUSION}

The study concludes that both motor therapy and sensorimotor therapy is beneficial in stroke rehabilitation, but sensorimotor therapy is more effective in improving the gross and fine motor skills of the upper limb of stroke patients. According to this study, sensorimotor training and motor training could have unique effects on different aspects of function, yet the impact on functional independence was similar. The afferent system provides critical input for the restoration of voluntary, fine motor movements. Normal and accurate sensorimotor feedback is critical to correct and improve motor efficiency and learning. This study suggests that sensory stimulation should not be used only as a reflex stimulus for a motor output; it should be used as part of the intervention. Once a patient demonstrates early voluntary control, then the sensorimotor training may be essential to enable recovery of fine motor skills. Motor training was associated with significant gains in fine motor skills, but sensorimotor training is associated with better performance of sensory discrimination and fine motor skills.

\section{CONFLICT OF INTEREST}

Authors declare no conflict of interest.

\section{REFERENCES}

1. Morreale, M., Marchione,T., Pilli, A., Antonella, L., Spallone, A., Pierelli, F., et al., early versus delayed rehabilitation treatment in hemiplegic patients with ischemic, Proprioceptive or cognitive approach. EUR J Physical Rehabilitation Medicine. 2016: 52(1): 81-89.

2. Chaturvedi, P., Singh, A. K., Kulshrehtha D., Maurya, P, K., Thacker, A. K., Effects of Proprioceptive neural facilitation exercise on upper extremity in the patients with acute stroke circulation; cardiovascular quality and outcome. 2016: 9 (supply 2): A -102.

3. Pollock, A., Baer, G. D., Langhorne, P., Valerie, M., Pomeroy. Physiotherapy treatment approach for stroke. 2008: 39(2): 838-899.

4. Cramer, S. C., Nelles, G., Benson, R. R., Kaplan, J. D., Parker, R. A., Kwong, K. K., et al., A functional mri study of subjects recovered from hemiparetic stroke. Stroke 2008: 28(3): 25182527.

5. Smajlovic, D. Z., Salihovic, D., Ibrahimic, O. C., Sinanovic, O. Characteristics of stroke in young adults in Tuzla Canton, Bosnia and Herzegovina. Coll Antropol. 2013: 37(4): 515-519.

6. Varona, J. F., Guerra, J. M., Bermejo, F., Molina, J. A., Gómez de la Cámara. Causes of ischemic stroke in young adults, and evolution of the etiological diagnosis over the long term. Eur Neurol. 2007: 57: 212-218.

7. Gowitze, B. A., Milner, M. Scientific basis of human movement. Baltimore; Williams \& Willian's 1988: 20: 858863.

8. Carr, Shepherd, Nordholm, Lynne. Motor assessment scale Evidence reviewed as of before 07-11-2010 Canadian partnership for stroke recovery 1985: 14: 281-289.
9. Victoria, G. D. The Proprioceptive neuromuscular facilitation stretching technique- A brief overview. 2013: 13(3); 623-626.

10. Martin, S. T., Kessler, M. Neurologic interventions for physical therapy. Netherlands: Elsevier Health Science: 2015.

11. Fugl Meyer, A. R., Jaskoh,L., Leyman, L., Olsson, S., Stegling, S. The post stroke hemiplegic patient. A method for evaluation of physical performance. Scand J Rehabil 2008: 18: 75- 84.

12. McCaskey., Micheal, A., Schuster-Amft, C., Writh, B., Eling, D. Effects of postural specific sensorimotor training in patients with chronic low back pain: study protocol for randomized controlled trial. Trails. 2015: 16: 571.

13. Twitchell, T. E., The restoration of motor function following hemiplegia in man. Brain. 1951: 74(4): 443-480.

14. Schneider, R., Gautier, J. C. Leg weakness due to stroke. Site of lesions, weakness patterns and causes. Brain. 2004: 117: 347-354.

15. Taniguchi, A., Li, Y., Kawana,Y., Asahi, M., Naito, Y., Shibata, M., et al., Case of ipsilateral monoparesis by lacunar infarction: a consideration of pathological mechanism. Brain Nerve. 2011: 63: 177-180.

16. Morris, M.E., Matyas, T.A., Bach, T.M., Goldie, P.A. The effect of electrogoniometer feedback on genu recurvatum in stroke. ArchPhys Med Rehabil 1990:73:348-354.

17. Olney, S.J., Griffin, M.P., Monga, T.N., McBride, I.D. Work and power in the gait of stroke patients. Arch Phys Med Rehabil 1991;72: 309-314.

18. Farber, S.D. Neurorehabilitation: a multisensory approach. Philadelphia:WB Saunders, 1982 :12(7):152-157.

19. Small, S. L., Buccino, G., Solodkin, A. The mirror neuron system and treatment of stroke. Dev. Psychobiol, 201254:293-310. 10.1002/dev.20504.

20. Brunner, I., Skouen, J.S., Hofstad, H., Strand, L.I., Becker, F., Sanders, A.M. Virtual reality training for upper extremity in subacute stroke (VIRTUES): De Bruyn et al. Trials 2018,19:242 Page 9 of 10 study protocol for a randomized controlled multicenter trial. BMC Neurol. 2014: 14:186.

21. Lewandowska, A. Therapeutic treatment according to the Bobath concept based on the international classification ICF in a patient after stroke-case report. 2018: 22(15): 665-674.

22. Mikolajewska, E. NDT bobath method in post stroke rehabilitation in adults aged 42-55 years preliminary; 2015: 98-104.

23. Victoria, G. D. The PNF (proprioceptive neuromuscular facilitation) stretching technique- A brief review; 2013; 13(3): 623-626.

24. Hsieh, I. P., Hsieh, C. L. Responsiveness of two upper extremity function instruments for stroke in patients receiving rehabilitation. Clini Rehabilitation. 2002:16: 617-624.

25. Platz, T., Pinkowski, C., Van wijck, F., In- ha, Kim., Bella, P., Johnson. G., et al., Reliability and validity of arm function assessment with standardized guidelines for the Fugl- Meyer test, action research arm test and box and block test. 2005: 19(4): 404-411. 